\title{
QUASI-INTERPOLATION AND A POSTERIORI ERROR ANALYSIS IN FINITE ELEMENT METHODS
}

\author{
Carsten Carstensen ${ }^{1}$
}

\begin{abstract}
One of the main tools in the proof of residual-based a posteriori error estimates is a quasiinterpolation operator due to Clément. We modify this operator in the setting of a partition of unity with the effect that the approximation error has a local average zero. This results in a new residualbased a posteriori error estimate with a volume contribution which is smaller than in the standard estimate. For an elliptic model problem, we discuss applications to conforming, nonconforming and mixed finite element methods.
\end{abstract}

AMS Subject Classification. 65N30, 65R20, 73C50.

Received: November 27, 1997. Revised: December 9, 1998.

\section{INTRODUCTION}

For a brief presentation of the quasi-interpolation operator $\mathcal{I}$ in a simplified setting, let us suppose in this introduction that $\mathcal{T}$ is a regular triangulation of a bounded Lipschitz domain $\Omega$ with a polygonal boundary $\Gamma$ in the plane. To indicate the improvements over [11], let us assume that $\mathcal{T}$ consists of triangles and parallelograms and that $\Gamma$ is split into a closed part $\Gamma_{D}$ of positive length for homogeneous Dirichlet boundary conditions and a remaining, possibly empty, part $\Gamma_{N}:=\Gamma \backslash \Gamma_{D}$. Let $\mathcal{N}$ denote the set of all nodes (i.e. the vertices of the elements) while $\mathcal{K}:=\mathcal{N} \backslash \Gamma_{D}$ denotes the set of free nodes.

For any node $z \in \mathcal{N}$, let $\varphi_{z}$ be the corresponding hat-function defined as the discrete function in the finite element space (excluding boundary conditions) that takes the value 1 at the node $z$ but vanishes at all other nodes. Hence, $\left(\varphi_{z} \mid z \in \mathcal{K}\right)$ is the nodal basis of the finite element space (including the homogeneous boundary conditions on $\left.\Gamma_{D}\right)$.

The family $\left(\varphi_{z} \mid z \in \mathcal{N}\right)$ is a partition of unity and this motivates our examination of arbitrary Lipschitz partitions of unity; the results then cover a large class of finite element methods, e.g., isoparametric finite elements, higher order elements in higher dimensions, or hanging nodes.

To define the quasi-interpolation operator $\mathcal{I}$ in the aforementioned two-dimensional setting, set $\psi:=\sum_{z \in \mathcal{K}} \varphi_{z}$ and, given any $f \in L^{1}(\Omega)$, define

$$
\mathcal{I} f:=\sum_{z \in \mathcal{K}} f_{z} \varphi_{z} \quad \text { for } f_{z}:=\frac{f, \varphi_{z} / \psi}{1, \varphi_{z}} \quad(z \in \mathcal{K})
$$

Keywords and phrases. A posteriori error estimates, adaptive algorithm, reliability, mixed finite element method, nonconforming finite element method.

1 Mathematisches Seminar, Christian-Albrechts-Universität zu Kiel, Ludewig-Meyn-Str. 4, 24098 Kiel, Germany.

e-mail: cc@numerik.uni-kiel.de 
where $(\cdot, \cdot)$ denotes the inner product in $L^{2}(\Omega)$. The error $f-\mathcal{I} f$ has a vanishing weighted average, i.e., for $f, g \in L^{2}(\Omega)$ and arbitrary $g_{z} \in \mathbb{R}, z \in \mathcal{K}$, there holds

$$
(f-\mathcal{I} f, g)=\sum_{z \in \mathcal{K}}\left(\frac{f \varphi_{z}}{\psi}-f_{z} \varphi_{z}, g-g_{z}\right) .
$$

As main consequences, one deduces the approximation and stability properties

$$
\begin{aligned}
& (f-\mathcal{I} f, g) \leq C\|\nabla f\|_{L^{2}(\Omega)}\left(\sum_{z \in \mathcal{K}} h_{z}^{2} \int_{\omega_{z}} \frac{\varphi_{z}\left|g-g_{z}\right|^{2}}{\psi} \mathrm{d} x\right)^{1 / 2}, \\
& \left\|h_{\mathcal{T}}^{-1}(f-\mathcal{I} f)\right\|_{L^{2}(\Omega)}+\|\nabla(f-\mathcal{I} f)\|_{L^{2}(\Omega)} \leq C\|\nabla f\|_{L^{2}(\Omega)} .
\end{aligned}
$$

The $\mathcal{T}$-piecewise constant weight $h_{\mathcal{T}}$ is the elementwise mesh-size, $h_{\mathcal{T}}=\operatorname{diam}(T)$ on $T \in \mathcal{T}$, $h_{z}$ denotes the diameter of the patch $\omega_{z}:=\cup\{T \in \mathcal{T} \mid z \in T\}$ of $z \in \mathcal{K}$, and the positive constant $C$ is independent of $f, g, h_{\mathcal{T}}$ and depends only on the shapes of the elements in $\mathcal{T}$. The improvement over the Clément operator is that $g_{z}$ allows a local reduction in (1.3). The disadvantage is that only linear convergence is provided through (1.4), while, for $f \in H^{2}(\Omega)$, the Clément quasi-interpolant is of second order.

One motivation for the above quasi-interpolation operator $\mathcal{I}$ was the question of dominating edge-contributions which is eventually solved by the results of this paper for arbitrary regular but unstructured meshes. It has been a conjecture in the engineering community for a long time that it suffices in residual-based error control to focus on the edge contributions such as a jump of the discrete stress along inner element edges.

For a very special mesh, Yu proved that the edge-contributions dominate the residual based standard a posteriori error estimates for piecewise polynomials of odd degree [21,22]. For unstructured grids, it was later shown in [11] that the volume contributions can be replaced by a term which is generically of higher order. For arbitrary meshes and with Dirichlet conditions, the results of this paper provide the estimate

$$
\|u-U\|_{W^{1,2}(\Omega)} \leq C\left\{\sum_{z \in \mathcal{K}} h_{z}^{2}\left\|f+\Delta_{\mathcal{T}} U-R_{z}\right\|_{L^{2}\left(\omega_{z}\right)}^{2}+\sum_{E \in \mathcal{E}} h_{E}\left\|\left[\partial U / \partial n_{E}\right]\right\|_{L^{2}(E)}^{2}\right\}^{1 / 2}
$$

for the exact solution $u \in H_{0}^{1}(\Omega)$ of a model problem $\Delta u+f=0$ with Galerkin solution $U$. The improvement over the standard error estimate is that $R_{z}$ is arbitrary in (1.5) (and vanishes in the standard estimate) and so lowers the volume terms in (1.5). To see this, suppose $U$ is $\mathcal{T}$-piecewise affine such that the $\mathcal{T}$-piecewise Laplacian $\Delta_{\mathcal{T}} U$ vanishes. Then $\left\|f+\Delta_{\mathcal{T}} U-R_{z}\right\|_{L^{2}\left(\omega_{z}\right)}^{2}$ is of higher order owing to a proper choice of $R_{z}$. If $f$ is smooth, a Poincaré inequality shows

$$
\|u-U\|_{W^{1,2}(\Omega)} \leq C\left\{\sum_{E \in \mathcal{E}} h_{E}\left\|\left[\partial U / \partial n_{E}\right]\right\|_{L^{2}(E)}^{2}\right\}^{1 / 2}+C\left\|h_{\mathcal{T}}^{2} \nabla f\right\|_{L^{2}(\Omega)},
$$

where the last term is of higher order. The remaining edge-contributions $\left\|\left[\partial U / \partial n_{E}\right]\right\|_{L^{2}(E)}^{2}$ describe the jump of the discrete stress $\nabla U$ across the inner element edge $E$ in normal direction $n_{E} ; \mathcal{E}$ denotes the set of all edges.

For nonconforming and mixed finite element methods, a similar technique shows that the volume contributions in the estimate can be sharpened. It is stressed that the improvements apply to any situation which usually Clément's paper [12] is quoted in, and so is useful for any (non-linear) partial differential equation in divergence form; $c f .[2-5,15,17,19]$.

The remaining part of the paper is organised as follows. A much more general setting for a Lipschitz partition of unity and the proposed interpolation operator are introduced in Section 2. Its approximation and stability properties are stated in Section 3 and proved in Section 4. To illustrate the standard setting the general frame is applied to, we specify the resulting sharpened a posteriori error estimate, first for conform piecewise polynomial 
finite element methods in Section 5, for nonconforming finite element methods such as Crouzeix-Raviart finite elements in Section 6, and finally for mixed finite elements in Section 7.

\section{WEAK INTERPOLATION OF LIPSCHITZ PARTITIONS OF UNITY}

Suppose that $\mathcal{K} \subseteq \mathcal{N}$ are finite (index) sets, and $\left(\varphi_{z} \mid z \in \mathcal{N}\right)$ is a Lipschitz partition of unity on $\Omega$, i.e.

$$
\begin{array}{cc}
\sum_{z \in \mathcal{N}} \varphi_{z}=1 & \text { on } \Omega, \\
0 \leq \varphi_{z}, \quad \varphi_{z} \text { Lipschitz continuous } & \text { on } \Omega \quad(z \in \mathcal{N})
\end{array}
$$

with $\operatorname{Lipschitz}$ constant $\operatorname{Lip}\left(\varphi_{z}\right)$. We assume that $\varphi_{z}$ is not identical zero such that

$$
\omega_{z}:=\left\{x \in \Omega \mid \varphi_{z}(x)>0\right\} \quad(z \in \mathcal{N})
$$

is non-void. We are interested in an interpolation operator

$$
\mathcal{I}: L^{1}(\Omega) \rightarrow \mathcal{S}
$$

where the linear space $\mathcal{S} \subseteq W^{1, \infty}(\Omega)$ involves only (some) Lipschitz functions of the family $\left(\varphi_{z} \mid z \in \mathcal{N}\right)$, namely

$$
\mathcal{S}:=\operatorname{span}\left\{\varphi_{z} \mid z \in \mathcal{K}\right\}
$$

Remark 2.1. The interpretation of $\mathcal{K} \not \equiv \mathcal{N}$ is that some of the functions $\varphi_{z}$ do not satisfy proper side restrictions such as (homogeneous) Dirichlet boundary conditions.

Definition 2.1. Set $\psi:=\sum_{z \in \mathcal{K}} \varphi_{z}$, and suppose that $\psi>0$ almost everywhere on $\Omega$. Then, let

$$
\psi_{z}:=\varphi_{z} / \psi \quad(z \in \mathcal{K})
$$

Remark 2.2. If $\psi$ is not strictly positive on $\Omega$, we have to reduce $\Omega$ to $\Omega^{\prime}:=\{x \in \Omega \mid \psi(x)>0\}$. Note that this is not a huge restriction, because any $\varphi \in \mathcal{S}$ satisfies $\varphi=0$ on $\Omega \backslash \bar{\Omega}^{\prime}$ (recall (2.2)). Thus, after we constructed some $\mathcal{I} f=\varphi$ in $\mathcal{S}$ on the restricted domain $\Omega^{\prime}$, we may extend $\varphi$ to $\Omega$ by zero on $\Omega \backslash \bar{\Omega}^{\prime}$ and so obtain a reasonable approximation $\mathcal{I} f$ in $\mathcal{S}$.

Proposition 2.1. $\left(\psi_{z} \mid z \in \mathcal{K}\right)$ is a partition of unity on $\Omega$, i.e. $\psi_{z}$ is non-negative and continuous with $1=$ $\sum_{z \in \mathcal{K}} \psi_{z}$ on $\Omega$.

Proof. Since $\varphi_{z}$ is non-negative, and all such functions sum up to $\psi$ (according to its definition), we have $1=\sum_{z \in \mathcal{K}} \psi_{z}$ almost everywhere on $\Omega$.

Remark 2.3. The functions $\psi_{z}$ are not necessarily Lipschitz continuous. For instance, let $\varphi_{1}(x)=x^{1+\epsilon} / 2$, $\varphi_{2}(x)=x / 2, \varphi_{3}=1-\varphi_{1}-\varphi_{2}$ for $0<x<1$ and $0<\epsilon<1$. If $\mathcal{K}=\{1,2\}$, then $\psi_{2}(x)=1 /\left(1+x^{\epsilon}\right)$, and so $\psi_{2}^{\prime}(x)=-\epsilon x^{\epsilon-1} /\left(1+x^{\epsilon}\right)^{2}$ is unbounded if $0<\epsilon \ll 1$.

Definition 2.2. Define $\mathcal{I}: L^{1}(\Omega) \rightarrow \mathcal{S}$ via

$$
\mathcal{I} f:=\sum_{z \in \mathcal{K}}\left(f, \psi_{z}\right) /\left(1, \varphi_{z}\right) \varphi_{z} \quad\left(f \in L^{1}(\Omega)\right)
$$

where $(\cdot, \cdot)$ denotes the inner product in $L^{2}(\Omega)$. 
Remark 2.4. The main orthogonality property mentioned in the abstract and the introduction is a simple consequence of the construction, namely, for $R, u \in L^{2}(\Omega)$, we have

$$
\int_{\Omega} R(u-\mathcal{I} u) \mathrm{d} x=\sum_{z \in \mathcal{K}} \int_{\Omega} \psi_{z}\left(R-R_{z}\right)\left(u-u_{z} \psi\right) \mathrm{d} x,
$$

where $R_{z} \in \mathbb{R}$ is arbitrary, while $u_{z}:=\left(u, \psi_{z}\right) /\left(1, \varphi_{z}\right)$.

Proof of (2.8). Recall that $\left(\psi_{z} \mid z \in \mathcal{K}\right)$ is a partition of unity. Thus,

$$
\begin{aligned}
\int_{\Omega} R(u-\mathcal{I} u) \mathrm{d} x & =\int_{\Omega} R\left(\sum_{z \in \mathcal{K}} u \psi_{z}-\sum_{\zeta \in \mathcal{K}} u_{\zeta} \varphi_{\zeta}\right) \mathrm{d} x \\
& =\sum_{z \in \mathcal{K}} \int_{\Omega} R\left(u-u_{z} \psi\right) \psi_{z} \mathrm{~d} x=\sum_{z \in \mathcal{K}} \int_{\Omega}\left(R-R_{z}\right)\left(u-u_{z} \psi\right) \psi_{z} \mathrm{~d} x
\end{aligned}
$$

because, owing to (2.6) and the definition of $u_{z}$,

$$
\int_{\Omega}\left(u-u_{z} \psi\right) \psi_{z} \mathrm{~d} x=0 .
$$

\section{Stability and approximation properties}

To include Dirichlet conditions, let $\Omega$ be a bounded Lipschitz domain in $\mathbb{R}^{n}$ with boundary $\partial \Omega$, and assume that there is some relatively closed part $\Gamma_{D}$ of $\partial \Omega$ such that the space (2.5) satisfies

$$
\begin{aligned}
\mathcal{S} & :=\operatorname{span}\left\{\varphi_{z} \mid z \in \mathcal{K}\right\} \subseteq W_{D}^{1, p}(\Omega):=\left\{u \in W^{1, p}(\Omega)|u|_{\Gamma_{D}}=0\right\} \\
\mathcal{K} & :=\left\{z \in \mathcal{N} \mid \varphi_{z} \in W_{D}^{1, p}(\Omega)\right\} .
\end{aligned}
$$

Theorem 3.1. Adopt notation from Section 2 and 3 for $1<p, q<\infty, 1 / p+1 / q=1$. Then, there exists a constant $C>0$ that depends only on $\Omega, \Gamma_{D}, p$ and the shape of the supports $\left(\omega_{z} \mid z \in \mathcal{K}\right)$, as well as on the shapes of $\left(\omega_{z} \cup \omega_{\zeta} \mid \zeta \in \mathcal{N} \backslash \mathcal{K}, z \in \mathcal{K}, \omega_{z} \cap \omega_{\zeta} \neq \emptyset\right.$ with $\psi \neq \equiv 1$ on $\left.\omega_{z}\right)$, the shape coefficients $\left(\int_{\omega_{z}} \varphi_{z} \mathrm{~d} x /\right.$ meas $\left.\left(\omega_{z}\right) \mid z \in \mathcal{K}\right)$, and on the overlap

$$
\begin{aligned}
& M_{1}:=\operatorname{esssup}_{x \in \Omega} \operatorname{card}\left\{z \in \mathcal{N} \mid \varphi_{z}(x)>0\right\}, \\
& M_{2}:=\max _{z \in \mathcal{N}} \operatorname{card}\left\{z \in \mathcal{K} \mid \omega_{z} \cap \omega_{\zeta} \neq \emptyset, \zeta \in \mathcal{N} \backslash \mathcal{K}\right\},
\end{aligned}
$$

but not on their sizes $h_{z}:=\operatorname{diam}\left(\omega_{z}\right)$, such that the following holds.

1. For all $u \in W_{D}^{1, p}(\Omega), R \in L^{q}(\Omega)$, and arbitrary $R_{z} \in \mathbb{R}, z \in \mathcal{K}$, there holds

$$
\int_{\Omega} R(u-\mathcal{I} u) \mathrm{d} x \leq C\|\nabla u\|_{L^{p}(\Omega)}\left(\sum_{z \in \mathcal{K}} h_{z}^{q} \int_{\Omega} \psi_{z}\left|R-R_{z}\right|^{q} \mathrm{~d} x\right)^{1 / q} .
$$

2. For all $u \in W_{D}^{1, p}(\Omega)$ there holds

$$
\left\|h^{-1}(u-\mathcal{I} u)\right\|_{L^{p}(\Omega)} \leq C\|\nabla u\|_{L^{p}(\Omega)},
$$


where $h(x):=\max \left\{h_{z} \mid \varphi_{z}(x)>0, z \in \mathcal{K}\right\}$.

3. There exists a constant $c>0$ that further depends on $\max _{z \in \mathcal{N}} h_{z} \operatorname{Lip}\left(\varphi_{z}\right)$ such that, for all $u \in W_{D}^{1, p}(\Omega)$,

$$
\|\nabla \mathcal{I} u\|_{L^{p}(\Omega)} \leq c\|\nabla u\|_{L^{p}(\Omega)} .
$$

It will be clear in Section 5, that Theorem 3.1 yields an estimate for the volumetric part of the residuals in the finite element analysis. To estimate edge contributions, we state a simple consequence in a general framework, which will be convenient in Sections 5-7.

Let $S$ be a finite union of Lipschitz surfaces, such that

$$
S \subseteq \underset{T \in \mathcal{T}}{\cup} \partial T
$$

where $\mathcal{T}$ is a finite set of pairwise disjoint, non-void Lipschitz domains in $\Omega$.

Theorem 3.2. There exists a constant $C>0$ that depends on the constants in (3.6-3.7), on

$$
M_{3}:=\operatorname{ess}_{\sup }{ }_{x \in \Omega}\left\{h(x) / h_{T} \mid x \in T \in \mathcal{T}\right\},
$$

and on the shape of the domains in $\mathcal{T}$, but not on their sizes such that, for all $J \in L^{q}(S)$ and all $u \in W_{D}^{1, p}(\Omega)$, $1<p, q<\infty, 1 / p+1 / q=1$,

$$
\int_{S} J(u-\mathcal{I} u) \mathrm{d} s \leq C\|\nabla u\|_{L^{p}(\Omega)}\left(\sum_{T \in \mathcal{T}} h_{T}\|J\|_{L^{q}(S \cap \partial T)}^{q}\right)^{1 / q} .
$$

The main results in Theorem 3.1 and 3.2 are applied in the following form.

Corollary 3.1. There exists a constant $C>0$ that depends on the constants in (3.6, 3.7, 3.10), such that

$$
\int_{\Omega} R(u-\mathcal{I} u) \mathrm{d} x+\int_{S} J(u-\mathcal{I} u) \mathrm{d} s \leq C\|\nabla u\|_{L^{p}(\Omega)}\left\{\sum_{z \in \mathcal{K}} h_{z}^{q}\left\|R-R_{z}\right\|_{L^{p}\left(\omega_{z}\right)}^{q}+\sum_{T \in \mathcal{T}} h_{T}\|J\|_{L^{q}(S \cap \partial T)}^{q}\right\}^{1 / q}
$$

holds for all $J \in L^{q}(S), R \in L^{q}(\Omega), u \in W_{D}^{1, p}(\Omega)$, and arbitrary $R_{z} \in \mathbb{R}$ for $z \in \mathcal{K}$.

\section{ProOFs}

In the proof of point 1 of Theorem 3.1, we are given $R \in L^{q}(\Omega)$ and $u \in W_{D}^{1, p}(\Omega)$, start as in (2.9), and obtain with Hölder's inequality

$$
\begin{aligned}
\int_{\Omega} R(u-\mathcal{I} u) \mathrm{d} x & =\sum_{z \in \mathcal{K}} \int_{\Omega}\left(R-R_{z}\right) \psi_{z}\left(u-u_{z} \psi\right) \mathrm{d} x \\
& \leq\left(\sum_{z \in \mathcal{K}} h_{z}^{q} \int_{\Omega} \psi_{z}\left|R-R_{z}\right|^{q} \mathrm{~d} x\right)^{1 / q}\left(\sum_{z \in \mathcal{K}} h_{z}^{-p} \int_{\Omega} \psi_{z}\left|u-u_{z} \psi\right|^{p} \mathrm{~d} x\right)^{1 / p} .
\end{aligned}
$$

Fix $z \in \mathcal{K}$ and consider $\int_{\Omega} \psi_{z}\left|u-u_{z} \psi\right|^{p} \mathrm{~d} x$. In the first case we assume $\psi_{z}=\varphi_{z}$, i.e. $\psi=1$ on $\omega_{z}$. Then, according to a Poincaré inequality, we claim the existence of a constant $c_{z}$ with

$$
\int_{\omega_{z}}\left|u-u_{z}\right|^{p} \mathrm{~d} x \leq c_{z} h_{z}^{p}\|\nabla u\|_{L^{p}\left(\omega_{z}\right)}^{p}
$$


We prove (4.2) by an indirect argument. If (4.2) was false, we could find a sequence $\left(u_{j}\right)$ in $W^{1, p}\left(\omega_{z}\right)$ with $\int_{\omega_{z}}\left|u_{j}-u_{j_{z}}\right|^{p} \mathrm{~d} x=1$ and $\left\|\nabla u_{j}\right\|_{L^{p}\left(\omega_{z}\right)} \leq 1 / j$. Hence, $\left(u_{j}\right)$ is bounded in $W^{1, p}\left(\omega_{z}\right)$, has a weakly converging subsequence $\left(u_{k}\right)$ which, according to compactness, is strongly convergent in $L^{p}\left(\omega_{z}\right)$. Since $\left(u_{k}\right)$ and $\left(\nabla u_{k}\right)$ converge strongly in $L^{p}\left(\omega_{z}\right)$, we conclude that $\left(u_{k}\right)$ converges strongly to some $u$ in $W^{1, p}\left(\omega_{z}\right)$. Since $\|\nabla \cdot\|_{L^{p}\left(\omega_{z}\right)}$ is lower semi-continuous, $\nabla u=0$, whence $u$ is constant. The sequence $\left(u_{j_{z}}\right)$ is convergent to $u_{z}=\left(u, \psi_{z}\right) /\left(1, \varphi_{z}\right)$, which equals the constant $u$ because $\psi_{z}=\varphi_{z}$. Thus, $\left(u_{k}-u_{k_{z}}\right)$ converges to zero in $L^{p}\left(\omega_{z}\right)$, which contradicts $\left\|u_{k}-u_{k_{z}}\right\|_{L^{p}\left(\omega_{z}\right)}=1$. This proves (4.2) in the first case. A simple scaling argument shows that $c_{z}$ is independent of $h_{z}:=\operatorname{diam}\left(\omega_{z}\right)$.

In the second case, we assume that $\psi \not \equiv 1$ on $\omega_{z}$. According to (2.1), there exists $\zeta \in \mathcal{N} \backslash \mathcal{K}$ with $\varphi_{\zeta} \not \equiv 0$ on $\omega_{z}$. By (3.2), $\varphi_{\zeta}>0$ on some part of $\Gamma_{D}$ with positive surface measure. Therefore, $\omega:=\omega_{z} \cup \omega_{\zeta}$ is open, connected, and $\partial \omega \cap \Gamma_{D}=: \gamma_{D}$ has positive surface measure. Thus there exists a Friedrichs' inequality for all $u \in W_{D}^{1, p}(\Omega)$, namely

$$
\int_{\omega}|u|^{p} \mathrm{~d} x \leq c_{z, \zeta} h_{z}^{p}\|\nabla u\|_{L^{p}(\omega)}^{p} \quad\left(u \in W_{D}^{1, p}(\omega)\right)
$$

where $W_{D}^{1, p}(\omega)=\left\{u \in W^{1, p}(\omega) \mid u=0\right.$ on $\left.\gamma_{D}\right\}$. A scaling argument again shows that the constant $c_{z, \zeta}$ in $(4.3)$ is independent of the size of $\omega$, but depends on its shape. (Note that $h_{z}:=\operatorname{diam}\left(\omega_{z}\right)$ is not the size of $\omega$, but $\operatorname{diam}(\omega) / h_{z}$ depends on the shape of $\omega$ and $\omega_{z}$ only.) With Hölder's inequality, $(a+b)^{p} \leq 2^{p / q}\left(a^{p}+b^{p}\right)$ for $a, b \geq 0$, we have

$$
\int_{\Omega} \psi_{z}\left|u-u_{z} \psi\right|^{p} \mathrm{~d} x \leq 2^{p / q} \int_{\omega_{z}}|u|^{p} \mathrm{~d} x+2^{p / q} \int_{\Omega} \psi_{z}\left|u_{z}\right|^{p} \psi^{p} \mathrm{~d} x
$$

According to $u_{z}:=\left(u, \psi_{z}\right) /\left(1, \varphi_{z}\right),(2.6)$, and Hölder's inequality,

$$
\begin{aligned}
\int_{\omega_{z}} \psi_{z} \psi^{p}\left|u_{z}\right|^{p} \mathrm{~d} x & =\int_{\omega_{z}} \varphi_{z} \psi^{p-1} \mathrm{~d} x\left|\left(u, \psi_{z}\right)\right|^{p} /\left(1, \varphi_{z}\right)^{p} \\
& \leq\|u\|_{L^{p}\left(\omega_{z}\right)}^{p}\left\|\psi_{z}\right\|_{L^{q}\left(\omega_{z}\right)}^{p}\left(1, \varphi_{z}\right)^{1-p} \leq\left(\operatorname{meas}\left(\omega_{z}\right) /\left(1, \varphi_{z}\right)\right)^{p-1}\|u\|_{L^{p}\left(\omega_{z}\right)}^{p} .
\end{aligned}
$$

Let $C_{1}, \ldots, C_{6}$ denote size-independent constants which depend only on the quantities and shapes mentioned in the theorem. Then, from (4.2-4.5), we conclude in all cases

$$
\int_{\Omega} \psi_{z}\left|u-u_{z} \psi\right|^{p} \mathrm{~d} x \leq C_{1} h_{z}^{p}\|\nabla u\|_{L^{p}\left(\omega_{z} \cup \omega_{\zeta}\right)}^{p}
$$

for all $z \in \mathcal{K}$ and possibly some $\zeta=\zeta(z) \in \mathcal{N} \backslash \mathcal{K}$ associated with $z$, such that $\omega=\omega_{z} \cup \omega_{\zeta}$. This implies

$$
\begin{aligned}
\sum_{z \in \mathcal{K}} h_{z}^{-p} \int_{\Omega} \psi_{z}\left|u-u_{z} \psi\right|^{p} \mathrm{~d} x & \leq C_{1} \sum_{z \in \mathcal{K}}\|\nabla u\|_{L^{p}\left(\omega_{z} \cup \omega_{\zeta(z)}\right)}^{p} \\
& =C_{1} \int_{\Omega}|\nabla u(x)|^{p} \operatorname{card}\left\{z \in \mathcal{K} \mid x \in \omega_{z} \cup \omega_{\zeta(z)}\right\} \mathrm{d} x \leq C_{2}\|\nabla u\|_{L^{p}(\Omega)}^{p} .
\end{aligned}
$$

(In the last estimate, we used $(3.3,3.4)$ to infer $C_{2} \leq C_{1}\left(M_{1}+M_{2}\right)$.) From $(4.7,4.1)$ we obtain $(3.5)$.

To prove 2 of Theorem 3.1, we consider $R=\operatorname{sign}(u-\mathcal{I} u) / h$ in the first part; $\operatorname{sign} v:=v /|v|$ if $|v|>0$, and sign $0:=0$. Choose $R_{z}=0$ and consider the right-hand side in (3.5). Since $|R| \leq 1 / h$, we obtain

$$
\sum_{z \in \mathcal{K}} h_{z}^{q} \int_{\Omega} \psi_{z}|R|^{q} \mathrm{~d} x \leq \int_{\Omega} \sum_{z \in \mathcal{K}}\left(h_{z} / h\right)^{q} \psi_{z} \mathrm{~d} x \leq 1
$$


and conclude (3.6).

To verify (3.7), we employ (2.1) and obtain $0=\sum_{z \in \mathcal{N}} \nabla \varphi_{z}$ almost everywhere in $\Omega$. Therefore, if $u_{z}:=0$ for $z \in \mathcal{N} \backslash \mathcal{K}$ and $u_{z}:=\left(\psi_{z}, u\right) /\left(\varphi_{z}, 1\right)$ for $z \in \mathcal{K}$,

$$
\begin{aligned}
\|\nabla \mathcal{I} u\|_{L^{p}(\Omega)}^{p} & =\int_{\Omega}\left|\sum_{z \in \mathcal{N}}\left(u_{z}-u(x)\right) \nabla \varphi_{z}(x)\right|^{p} \mathrm{~d} x \\
& \leq M_{1}^{p / q} \sum_{z \in \mathcal{N}} \int_{\omega_{z}}\left|u_{z}-u(x)\right|^{p} \operatorname{Lip}\left(\varphi_{z}\right)^{p} \mathrm{~d} x .
\end{aligned}
$$

In the last estimate we noticed that, for almost all $x$ in $\Omega, \nabla \varphi_{z}(x) \neq 0$ for at most $M_{1}$ parameters $z$ in $\mathcal{N}$, so that we could apply Hölder's inequality in $\mathbb{R}^{M_{1}}$. (In $(4.9)$, Lip $\left(\varphi_{z}\right)$ denotes the Lipschitz constant of $\varphi_{z}$.) In case $z \in \mathcal{K}$ and $\varphi_{z} \equiv \psi_{z},(4.2)$ yields

$$
\int_{\omega_{z}}\left|u_{z}-u\right|^{p} \mathrm{~d} x \leq C_{1} h_{z}^{p}\|\nabla u\|_{L^{p}\left(\omega_{z}\right)}^{p}
$$

In case that $z \in \mathcal{K}$ and $\varphi_{z} \not \equiv \psi_{z}$, we argue as in (4.4-4.6) and obtain

$$
\int_{\omega_{z}}\left|u_{z}-u\right|^{p} \mathrm{~d} x \leq C_{3} h_{z}^{p}\|\nabla u\|_{L^{p}\left(\omega_{z} \cup \omega_{\zeta}(z)\right)}^{p} .
$$

(Note that now there is a factor $\psi_{z}$ missing, which leads to a slight modification and so a different constant $C_{3}$.) It remains the case $z \in \mathcal{N}$, where $u_{z}=0$ and $z \notin \mathcal{K}$, i.e. $\varphi_{z}>0$ on some part of $\Gamma_{D}$ with positive surface measure. As in (4.3), we infer from Friedrichs' inequality that

$$
\int_{\omega_{z}}|u|^{p} \mathrm{~d} x \leq C_{4} h_{z}^{p}\|\nabla u\|_{L^{p}\left(\omega_{z}\right)}^{p}
$$

From (4.9-4.12) we derive

$$
\|\nabla \mathcal{I} u\|_{L^{p}(\Omega)}^{p} \leq C_{5} \sum_{z \in \mathcal{N}} \operatorname{Lip}\left(\varphi_{z}\right)^{p} h_{z}^{p}\|\nabla u\|_{L^{p}\left(\omega_{z} \cup \omega_{\zeta(z)}\right)}^{p} .
$$

(Here, $\zeta(z)$ is given in $(4.11)$, and $\zeta(z)=z$ in $(4.10,4.12)$.) Let $C_{6}:=\max _{z \in \mathcal{N}} h_{z} \operatorname{Lip}\left(\varphi_{z}\right)$. Then (4.13) yields

$$
\|\nabla \mathcal{I} u\|_{L^{p}(\Omega)}^{p} \leq C_{5} C_{6}^{p} \sum_{z \in \mathcal{N}}\|\nabla u\|_{L^{p}\left(\omega_{z} \cup \omega_{\zeta}(z)\right)}^{p} .
$$

In the last step we argue as in $(4.7)$, and employ $(3.3,3.4)$ to conclude $(3.7)$.

In order to prove Theorem 3.2, we quote a trace theorem which is utilised frequently in the literature (e.g., in $[6,9,12])$. A proof is sketched only for completeness.

Proposition 4.1. If $\omega$ is a bounded Lipschitz domain, then there exists a constant $c(\omega)$ which depends only on the shape of the domain $\omega$ but not on its size $\operatorname{diam}(\omega)$, such that, for all $f \in W^{1, p}(\omega)$,

$$
\|f\|_{L^{p}(\partial \omega)}^{p} \leq c(\omega)\left\{\|f\|_{L^{p}(\omega)}^{p} / \operatorname{diam}(\omega)+\|\nabla f\|_{L^{p}(\omega)}^{p} \operatorname{diam}(\omega)^{p-1}\right\}
$$

Proof. According to trace inequalities in Sobolev spaces, we have

$$
\|f\|_{L^{p}(\partial \omega)}^{p} \leq c_{1}\|f\|_{W^{1-1 / p, p}(\partial \omega)}^{p} \leq c_{2}\|f\|_{W^{1, p}(\omega)}^{p}
$$


for $\omega$-depending constants $c(\omega)$. To see that $c(\omega)$ is diam $(\omega)$-independent, we employ a scaling argument and verify that $\|f\|_{L^{p}(\partial \omega)},\|f\|_{L^{p}(\omega)} / \operatorname{diam}(\omega)^{1 / p}$ and $\|\nabla f\|_{L^{p}(\omega)} \operatorname{diam}(\omega)^{1-1 / p}$ scale with a joint factor $\operatorname{diam}(\omega)^{(d-1) / p}$. From this we conclude the assertion.

Proof of Theorem 3.2. Let us write $h_{z}:=\operatorname{diam}\left(\omega_{z}\right), z \in \mathcal{N}$, and $h_{T}:=\operatorname{diam}(T), T \in \mathcal{T}$. According to (3.8), we have

$$
\int_{S} J(u-\mathcal{I} u) \mathrm{d} s \leq \sum_{T \in \mathcal{T}} \int_{S \cap \partial T}|J||u-\mathcal{I} u| \mathrm{d} s
$$

and with Hölder's inequality and (4.15)

$$
\begin{aligned}
\int_{S} J(u-\mathcal{I} u) \mathrm{d} s & \leq 2^{p / q} \sum_{T \in \mathcal{T}} c(T)\|J\|_{L^{q}(S \cap \partial T)}\left(h_{T}^{-1 / p}\|u-\mathcal{I} u\|_{L^{p}(T)}+h_{T}^{1-1 / p}\|\nabla(u-\mathcal{I} u)\|_{L^{p}(T)}\right) \\
& \leq C_{7}\left(\sum_{T \in \mathcal{T}} h_{T}\|J\|_{L^{q}(S \cap \partial T)}^{q}\right)^{1 / q}\left(\sum_{T \in \mathcal{T}}\left(\|u-\mathcal{I} u\|_{L^{p}(T)} / h_{T}+\|\nabla(u-\mathcal{I} u)\|_{L^{p}(T)}\right)^{p}\right)^{1 / p} .
\end{aligned}
$$

From $(3.6,3.7,3.9)$ we conclude

$$
\begin{aligned}
\sum_{T \in \mathcal{T}}\left(\|u-\mathcal{I} u\|_{L^{p}(T)} / h_{T}+\| \nabla(u\right. & \left.-\mathcal{I} u) \|_{L^{p}(T)}\right)^{p} \\
& \leq \sum_{T \in \mathcal{T}}\left(M_{3}\left\|h^{-1}(u-\mathcal{I} u)\right\|_{L^{p}(T)}+\|\nabla(u-\mathcal{I} u)\|_{L^{p}(T)}\right)^{p} \\
& \leq \sum_{T \in \mathcal{T}}\left(2 M_{3} C_{8}\right)^{p}\|\nabla u\|_{L^{p}(T)}^{p}=\left(2 M_{3} C_{8}\right)^{p}\|\nabla u\|_{L^{p}(\Omega)}^{p}
\end{aligned}
$$

(In the last identity, we used that $\cup \mathcal{T} \subseteq \Omega$ without any overlap.) From $(4.18,4.19)$ we obtain $(3.10)$.

Remark 4.1. The main improvement over [11] is that volume contributions near the Dirichlet boundary are, compared to the remaining parts, not treated differently.

\section{Application to COnform finite element methods}

In this section, we focus on finite element methods that include $P_{1}$ - and $Q_{1}$-finite elements which are continuous and satisfy homogeneous Dirichlet boundary conditions on $\Gamma_{D}$ exactly. To describe the discrete space $\mathcal{S} \subseteq W_{D}^{1, p}(\Omega)$, we firstly recall the definition of a regular triangulation in the sense of Ciarlet $[6,13]$.

Definition 5.1 (Regular triangulation). A regular triangulation $\mathcal{T}$ of the bounded Lipschitz domain $\Omega \subseteq \mathbb{R}^{d}$, $d=1,2,3$, with piecewise affine Lipschitz boundary $\Gamma=\partial \Omega=\Gamma_{D} \cup \Gamma_{N}$, consists of a finite number of closed subsets of $\bar{\Omega}$, that cover $\bar{\Omega}=\cup \mathcal{T}$. Each element $T \in \mathcal{T}$ includes an open ball $B(x, r) \subseteq T$ with maximal positive radius $r=\rho_{T}$ around some $x$, and is either an interval $T=\operatorname{conv}\{a, b\}$ if $d=1$, a triangle $T=\operatorname{conv}\{a, b, c\}$ or a parallelogram $T=\operatorname{conv}\{a, b, c, d\}$ if $d=2$, or a tetrahedron $T=\operatorname{conv}\{a, b, c, d\}$ or a parallelepiped $T=\operatorname{conv}\{a, b, c, d, e, f, g, h\}$ if $d=3$. The extremal points $a, \ldots, h$ are called vertices, and the faces $E \subseteq \partial T$ such as $E=a$ if $d=1, E=\operatorname{conv}\{a, b\}$ if $d=2$, or $E=\operatorname{conv}\{a, b, c\}$ resp. a parallelogram $\operatorname{conv}\{a, b, c, d\}$ if $d=3$ are called edges. The set of all vertices and all edges appearing for some $T$ in $\mathcal{T}$ are denoted as $\mathcal{N}$ and $\mathcal{E}$. Two distinct and intersecting $T_{1}$ and $T_{2}$ share either an entire edge, an interval or a vertex. Each edge $E \in \mathcal{E}$ on the boundary $\Gamma$ belongs either to $\Gamma_{D}$ or to $\bar{\Gamma}_{N}$. Finally, $\mathcal{K}:=\mathcal{N} \backslash \Gamma_{D}$ denotes the set of free nodes.

The lowest order conform finite element space with respect to $\mathcal{T}$ is denoted as $\mathcal{S}$. 
Definition $5.2\left(\mathcal{S}, \mathcal{S}_{D}\right)$. For $T \in \mathcal{T}$, let $P_{T}:=\mathcal{P}_{1}(T)$ if $d=1$, or if $T$ is a triangle and $d=2$, or if $T$ is a tetrahedron and $d=3$; let $P_{T}:=\mathcal{Q}_{1}(T)$ if $T$ is a parallelogram and $d=2$, or a parallelepiped and $d=3$. Here, $\mathcal{P}_{k}(K)$ [resp. $\left.\mathcal{Q}_{k}(K)\right]$ denotes the set of algebraic polynomials in $d$ variables on $K$ of total [resp. partial] degree $\leq k$. The (nonconforming) discrete space $\mathcal{L}_{1}(\mathcal{T})$ is the set of all $U \in L^{\infty}(\Omega)$ with restrictions in $P_{T}$, i.e., $\left.U\right|_{T} \in P_{T}$ for all $T$ in $\mathcal{T}$. Then, let $\mathcal{S}:=\mathcal{L}_{1}(\mathcal{T}) \cap W^{1, p}(\Omega)$ and $\mathcal{S}_{D}:=\mathcal{L}_{1}(\mathcal{T}) \cap W_{D}^{1, p}(\Omega)$. For each $z \in \mathcal{N}$, let $\varphi_{z}$ denote the discrete function in $\mathcal{S}$ that satisfies $\varphi_{z}(x)=0$ if $x \in \mathcal{N} \backslash\{z\}$ and $\varphi_{z}(z)=1 ;\left(\varphi_{z} \mid z \in \mathcal{K}\right)$ is the nodal basis of $\mathcal{S}_{D}$.

To illustrate the estimates of Section 3, suppose that we aim to approximate an unknown stress field $\sigma \in$ $L^{q}(\Omega)^{d}$ that satisfies

$$
\begin{aligned}
\operatorname{div} \sigma+f & =0 \quad \text { in } \Omega, \\
\sigma \cdot n & =g \text { on } \Gamma_{N},
\end{aligned}
$$

for some given $f \in L^{q}(\Omega)$ and $g \in L^{q}\left(\Gamma_{N}\right)$. (In (5.1), $\operatorname{div} \sigma$ is the distributional divergence which, by (5.1), is regular and then, (5.2) can be defined in a weak form via integration by parts formulae.) The weak form of $(5.1,5.2)$ is obtained straight forwardly by applying an integration by parts, and we suppose that we calculated some $\Sigma \in L^{q}(\Omega)$ that satisfies the weak form of $(5.1,5.2)$ for all test functions in $\mathcal{S}_{D}$, i.e., we suppose we are given $\Sigma$ with

$$
\int_{\Omega} \Sigma \cdot \nabla V \mathrm{~d} x=\int_{\Omega} f V \mathrm{~d} x+\int_{\Gamma_{N}} g V \mathrm{~d} s \quad\left(V \in \mathcal{S}_{D}\right)
$$

and $\left.\Sigma\right|_{T} \in W^{1, p}(T)^{d}$ for each $T \in \mathcal{T}$. We regard $\Sigma$ as an equilibrium approximation to $\sigma$ and obtain an a posteriori error estimate for

$$
\|\operatorname{div}(\sigma-\Sigma)\|_{W_{D}^{1, p}(\Omega)^{*}}:=\sup _{W \in W_{D}^{1, p}(\Omega) \backslash\{0\}} \int_{\Omega}(\sigma-\Sigma) \cdot \nabla W \mathrm{~d} x /\|\nabla W\|_{L^{p}(\Omega)} .
$$

To describe the computable upper bound, we define $J \in L^{q}(\bigcup \mathcal{E})$ for each edge $E \in \mathcal{E}$ by

$$
\left.J\right|_{E}= \begin{cases}\left(\left.\Sigma\right|_{T_{2}}-\left.\Sigma\right|_{T_{1}}\right) \cdot n_{E} & \text { if } E=T_{2} \cap T_{1} \nsubseteq \Gamma, \\ g-\left.\Sigma\right|_{T_{1}} \cdot n & \text { if } E \subseteq \bar{\Gamma}_{N} \cap T_{1}, \\ 0 & \text { if } E \subseteq \Gamma_{D} .\end{cases}
$$

In the first case, $T_{1}, T_{2} \in \mathcal{T}$ share the edge $E$ with unit normal $n_{E}$ on $E$, which is the outer normal of $T_{1}$, in the second case $E$ is an edge of $T_{1}$ on the boundary $\bar{\Gamma}_{N}$, where $n$ denotes the outer unit normal. Finally, let $h_{T}:=\operatorname{diam}(T)$ for $T \in \mathcal{T}, h_{E}:=\operatorname{diam}(E)$ for $E \in \mathcal{E}$, and $h_{z}:=\operatorname{diam}\left(\omega_{z}\right)$ for $z \in \mathcal{N}$. Let $\operatorname{div} \mathcal{T} \operatorname{denote}$ the $\mathcal{T}$-piecewise divergence, e.g., $\left.(\operatorname{div} \mathcal{T} \Sigma)\right|_{T}:=\operatorname{div}\left(\left.\Sigma\right|_{T}\right)$ for all $T \in \mathcal{T}$.

Theorem 5.1. There exists a constant $C>0$ which depends on $\max _{T \in \mathcal{T}} h_{T} / \rho_{T}, \Omega$, and $\Gamma_{D}$ such that, for all $R_{z} \in \mathbb{R}, z \in \mathcal{K}$, we have

$$
\|\operatorname{div}(\sigma-\Sigma)\|_{W_{D}^{1, p}(\Omega)^{*}} \leq C\left\{\sum_{z \in \mathcal{K}} h_{z}^{q}\left\|f+\operatorname{div} \mathcal{T}^{\Sigma}-R_{z}\right\|_{L^{q}\left(\omega_{z}\right)}^{q}+\sum_{E \in \mathcal{E}} h_{E}\|J\|_{L^{q}(E)}^{q}\right\}^{1 / q} .
$$

Proof of Theorem 5.1. By integration by parts, we obtain from (5.1-5.3) that

$$
\begin{aligned}
\int_{\Omega}(\sigma-\Sigma) \cdot \nabla w \mathrm{~d} x & =\int_{\Omega}(\sigma-\Sigma) \cdot \nabla(w-\mathcal{I} w) \mathrm{d} x \\
& =\int_{\Omega} R(w-\mathcal{I} w) \mathrm{d} x+\int_{\bigcup \mathcal{E}} J(w-\mathcal{I} w) \mathrm{d} s
\end{aligned}
$$




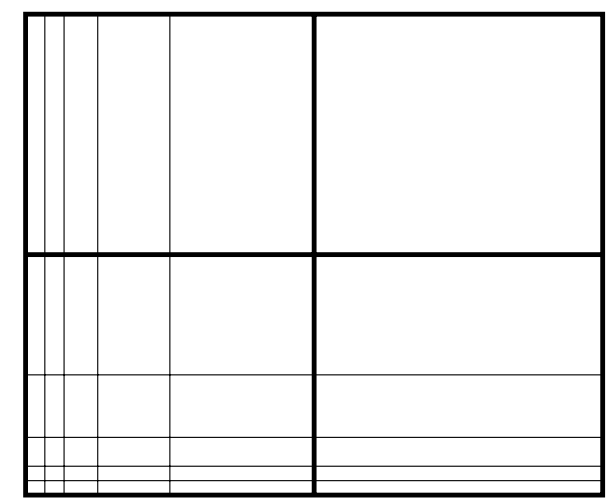

FiguRE 1. Fine (in thin lines) and shape-regular coarse triangulation $\mathcal{T}$ (in thick lines)

where $R:=f+\operatorname{div} \mathcal{T} \Sigma$, and $J$ is given in (5.5). Thus, (5.6) is a direct consequence of (5.7) and Corollary 3.1.

\section{Remarks 5.1.}

1. The estimate (5.6) yields error estimates for the displacement fields if we suppose a smooth uniformly monotonous stress strain relation, i.e., if there is a smooth $\mathcal{A}: W_{D}^{1, p}(\Omega) \rightarrow L^{q}(\Omega)^{d}$ with

$$
\alpha\|u-v\|_{W^{1, p}(\Omega)}^{p} \leq \int_{\Omega}(\mathcal{A}(u)-\mathcal{A}(v)) \cdot(\nabla u-\nabla v) \mathrm{d} x \quad\left(u, v \in W_{D}^{1, p}(\Omega)\right) .
$$

Then, if $\sigma:=\mathcal{A}(u)$ and $\Sigma:=\mathcal{A}(U)$, we obtain

$$
\begin{aligned}
\alpha\|u-U\|_{W^{1, p}(\Omega)}^{p} & \leq \int_{\Omega}(\sigma-\Sigma) \cdot \nabla(u-U) \mathrm{d} x \\
& \leq\|\operatorname{div}(\sigma-\Sigma)\|_{W_{D}^{1, p}(\Omega)^{*}}\|u-U\|_{W^{1, p}(\Omega)}
\end{aligned}
$$

and whence, according to (5.6), an a posteriori error estimate

$$
\|u-U\|_{W^{1, p}(\Omega)}^{p} \leq(C / \alpha)^{q}\left\{\sum_{z \in \mathcal{K}} h_{z}^{q}\left\|f+\operatorname{div}_{\mathcal{T}} \Sigma-R_{z}\right\|_{L^{q}\left(\omega_{z}\right)}^{q}+\sum_{E \in \mathcal{E}} h_{E}\|J\|_{L^{q}(E)}^{q}\right\} .
$$

From the above arguments we deduce the a posteriori error estimate (1.5) discussed in the introduction.

2. Reverse inequalities hold under some regularity conditions on the data, see [19].

3. Although Theorem 5.1 is formulated for scalar problems only, it applies straight forwardly componentwise to vectorial problems such as problems in linear and (partly) nonlinear elasticity.

4. It is stressed that we only need (5.3) to hold for the lowest order conform space $\mathcal{S}_{D}$. Of course the test finite element space may be much larger, and so our analysis includes $h p$-versions of the finite element method as well.

5. For thin and long elements $(d \geq 2)$, the constant $C$ in Theorem 5.1 is expected to deteriorate [9, 10]. It is stressed that we only need a shape-regular triangulation for the partition of unity, and so the fine triangulation may include very thin and relatively long elements on the price of error information on the coarse grid only. This situation is depicted in Figure 1, where a fine mesh is used near an edge singularity, while the coarse mesh (described by thick lines in Fig. 1) indicates the triangulation $\mathcal{T}$, to which (5.3) and thus (5.6) applies. 
6. Hanging nodes may be included in the analysis, since there exists a partition of unity in this case as well.

7. Finally, isoparametric finite element methods can be treated similarly. The analysis is straight forward. However, in practise, isoparametric elements are utilised for curved boundaries and so the triangulation does not exactly match the boundary. This causes further difficulties that lie beyond the scope of this paper.

8. The assertion justifies, to some extend, the averaging technique for a posteriori error control (cf. [3] for a similar statement and [19] for averaging estimates).

\section{Application to NONCONFORMing Finite ElEMEnt Methods}

In this section, we consider a linear model problem with the exact solution $u \in H_{0}^{1}(\Omega)$ of

$$
\sigma=A \nabla u \quad \text { and } \quad \operatorname{div} \sigma+f=0 \quad \text { in } \Omega .
$$

The linear and bounded operator $A: L^{2}(\Omega)^{2} \rightarrow L^{2}(\Omega)^{2}$ is supposed to be self-adjoint and uniformly elliptic, and we are given $f \in L^{2}(\Omega)$.

For a simple notation, we describe the two-dimensional situation only and suppose the nonconforming Galerkin solution $U$ belongs to

$$
\begin{aligned}
\mathcal{S}_{N C} & :=\left\{u \in H_{D}^{1}(\mathcal{T}) \mid \forall E \in \mathcal{E}, \int_{E \backslash \Gamma_{D}}[U] \mathrm{d} s=0\right\}, \\
H_{D}^{1}(\mathcal{T}) & :=\left\{u \in L^{2}(\Omega) \mid D_{\mathcal{T}} u \in L^{2}(\Omega) \text { and } \forall E \in \mathcal{E}, \int_{E \cap \Gamma_{D}} u \mathrm{~d} s=0\right\},
\end{aligned}
$$

where, for each edge $E \in \mathcal{E},[U]$ denotes the jump across $E$, i.e.,

$$
\left.[U]\right|_{E}:= \begin{cases}\left.\left(\left.U\right|_{T_{2}}-\left.U\right|_{T_{1}}\right)\right|_{E} & \text { if } E=T_{2} \cap T_{1}, \\ \left.U\right|_{E} & \text { if } E \subseteq \Gamma_{D} \cup \Gamma_{N} .\end{cases}
$$

(In the first case, $T_{1}, T_{2} \in \mathcal{T}$ and $\left.U\right|_{T_{j}}$ is the restriction of $U$ onto $T_{j}$, while $\left.\left(\left.U\right|_{T_{j}}\right)\right|_{E}$ is its trace on $E \subseteq \partial T_{j}$.) In (6.3), $D_{\mathcal{T}}$ denotes the $\mathcal{T}$-piecewise gradient, i.e., $\left.\left(D_{\mathcal{T}} U\right)\right|_{T}=\nabla\left(\left.u\right|_{T}\right)$ for each $T \in \mathcal{T}$.

Suppose that $U \in \mathcal{S}_{N C}$ satisfies $\operatorname{div} \mathcal{T} A D_{\mathcal{T}} U \in L^{2}(\Omega)$ and, as part of the Galerkin conditions,

$$
\int_{\Omega}\left(A D_{\mathcal{T}} U\right) \cdot \nabla V \mathrm{~d} x=\int_{\Omega} f V \mathrm{~d} x \quad\left(V \in \mathcal{S}_{D}\right) .
$$

The following result sharpens [14] (where $R_{z}=0, z \in \mathcal{K}$ ).

Theorem 6.1. There exists a constant $C>0$ which depends on $A, \max _{T \in \mathcal{T}} h_{T} / \rho_{T}$, and $\Omega$ such that, for all $R_{z} \in \mathbb{R}, z \in \mathcal{K}$, we have

$$
\begin{aligned}
\left\|D_{\mathcal{T}}(u-U)\right\|_{L^{2}(\Omega)} \leq & C\left\{\sum_{z \in \mathcal{K}} h_{z}^{2}\left\|f+\operatorname{div} \mathcal{\mathcal { T }} A D_{\mathcal{T}} U-R_{z}\right\|_{L^{2}\left(\omega_{z}\right)}^{2}\right. \\
& \left.+\sum_{E \in \mathcal{E}} h_{E}\left(\|J\|_{L^{2}(E)}^{2}+\|[\partial U / \partial s]\|_{L^{2}(E)}^{2}\right)\right\}^{1 / 2} .
\end{aligned}
$$

Here, $J$ is defined through $\Sigma:=A D_{\mathcal{T}} U$ in (5.5) and, $[\partial U / \partial s]$ is the jump of the tangential derivatives across an element edge $E$ where $U$ is extended by zero outside of $\Omega$ to define $[\partial U / \partial s]$ on $\Gamma$ as well. 


\section{Remarks 6.1.}

1. The assumption $\mathcal{S}_{D} \subseteq \mathcal{S}_{N C}$ is satisfied for triangulations that involve Courant triangles. For parallelograms, $\mathcal{S}_{D} \subseteq \mathcal{S}_{N C}$ requires higher order polynomials in $\mathcal{S}_{N C}$ to guarantee that mixed products (such as $x y$ ) belong to the shape functions in $\mathcal{S}_{N C}$ (and then include the $Q_{1}$ finite element).

2. If $A D_{\mathcal{T}} U$ is $\mathcal{T}$-piecewise constant, the volume contribution $\left\|f+\operatorname{div}_{\mathcal{T}} A D_{\mathcal{T}} U-R_{z}\right\|_{L^{2}\left(\omega_{z}\right)}^{2}$ is of higher order, i.e., if $f \in H^{1}(\Omega)$, we have as in the introduction

$$
\left\|D_{\mathcal{T}}(u-U)\right\|_{L^{2}(\Omega)} \leq C\left\{\sum_{E \in \mathcal{E}} h_{E}\left(\|J\|_{L^{2}(E)}^{2}+\|[\partial U / \partial s]\|_{L^{2}(E)}^{2}\right)\right\}^{1 / 2}+C\left\|h_{\mathcal{T}}^{2} \nabla f\right\|_{L^{2}(\Omega)} .
$$

Thus, the edge contributions generically dominate the a posteriori error estimate.

3. Reverse inequalities hold under some regularity assumptions on the data [14].

4. The assumptions allow a mixture of conforming and nonconforming elements, and a mixture of triangles and parallelograms in the plane.

5. Instead of the volume contribution, we could neglect the edge term $\left[\Sigma n_{E}\right]$ [20]. To see this, suppose that $\left.\left[\Sigma n_{E}\right]\right|_{E}$ is constant for each $E$, and that there exists $V_{E} \in H_{D}^{1}(\mathcal{T})$, such that

$$
\int_{\Omega} \Sigma \cdot D_{\mathcal{T}} V_{E} \mathrm{~d} x+\int_{\Omega} \operatorname{div} \sigma V_{E} \mathrm{~d} x=0
$$

and $\left.\int_{E^{\prime}} V_{E}\right|_{T} \mathrm{~d} s=0$ for all $E \in \mathcal{E} \backslash\left\{E^{\prime}\right\}$ and $T \in \mathcal{T}$, but $\left.V_{E}\right|_{E} \equiv 1$ (in particular, $V_{E}$ is continuous at $E$ ). Then, an elementwise integration by parts shows

$$
\int_{\Omega} V_{E} \operatorname{div} \mathcal{T}(\sigma-\Sigma) \mathrm{d} x=\int_{E}\left[\Sigma n_{E}\right] \mathrm{d} s
$$

because $\int_{E^{\prime}} V_{E} \mathrm{~d} s=0$, and $\Sigma n_{E}$ is constant. Hence, the constant $\left.\left[\Sigma n_{E}\right]\right|_{E}$ satisfies

$$
h_{E}\left\|\left[\Sigma n_{E}\right]\right\|_{L^{2}(E)}^{2} \leq h_{E} \operatorname{meas}\left(\omega_{E}\right)\left\|f-\operatorname{div} \mathcal{T}^{\Sigma}\right\|_{L^{2}\left(\omega_{E}\right)}^{2} .
$$

Here we assumed $\left\|V_{E}\right\|_{L^{\infty}(\Omega)} \leq 1$, and $\omega_{E}=\operatorname{supp} V_{E}$ is a neighbourhood of $E$. For Crouzeix-Raviart finite elements, this shows that the volume residuals dominate the edge terms. Hence, our theorem complements this property and states conversely, that the edge terms dominate.

Proof of Theorem 6.1. Set $\sigma:=A \nabla u$ and $e:=u-U$. Then, according to (6.5), we have with some ellipticity constant $\alpha$ of $A$, for $W$ in $\mathcal{S}_{D}$,

$$
\alpha\left\|D_{\mathcal{T}} e\right\|_{L^{2}(\Omega)}^{2} \leq \int_{\Omega}(\sigma-\Sigma) \cdot D_{\mathcal{T}} e \mathrm{~d} x=\int_{\Omega}(\sigma-\Sigma) \cdot D_{\mathcal{T}}(e-W) \mathrm{d} x .
$$

To adopt the results in Section 3 we decompose $D_{\mathcal{T}}(e-W) \in L^{2}(\Omega)^{2}$. Let $a \in H_{0}^{1}(\Omega)$ denote the unique (weak) solution to

$$
\operatorname{div}(A \nabla a)=\operatorname{div} \Sigma \text { in } \Omega
$$

and so $T:=\Sigma-A \nabla a$ satisfies

$$
\int_{\Omega} T \cdot \nabla \eta \mathrm{d} x=0 \quad\left(\eta \in H_{0}^{1}(\Omega)\right) .
$$


Then we choose $W:=\mathcal{I}(u-a) \in \mathcal{S}_{D}$ and recast the right-hand side of (6.11) as

$$
\int_{\Omega}(\sigma-\Sigma) \cdot \nabla(u-a-\mathcal{I}(u-a)) \mathrm{d} x-\int_{\Omega}\left(A^{-1} T\right) \cdot(\sigma-\Sigma) \mathrm{d} x .
$$

The first term can be rewritten according to an elementwise integration by parts and then estimated with (3.11). The calculation is as in (5.7), and so we neglect the details, but stress that the improved estimates apply here.

The second term in (6.14) involves Helmholtz decomposition as in $[1,8,14]$. Notice that

$$
\left\|A^{-1 / 2} T\right\|_{L^{2}(\Omega)}=\min _{b \in H_{0}^{1}(\Omega)}\left\|A^{-1 / 2} \Sigma-A^{1 / 2} \nabla b\right\|_{L^{2}(\Omega)} \leq\left\|A^{-1 / 2}(\Sigma-\sigma)\right\|_{L^{2}(\Omega)} .
$$

Because $T$ is divergence free in the simply connected domain $\Omega$, there exists a stream function $\phi \in H^{1}(\Omega)$ to $T$, i.e., $T=\operatorname{Curl} \phi:=\left(\partial \Phi / \partial x_{2},-\partial \Phi / \partial x_{1}\right)[16,18]$. This shows

$$
-\int_{\Omega}\left(A^{-1} T\right) \cdot(\sigma-\Sigma) \mathrm{d} x=\int_{\Omega} D_{\mathcal{T}} U \cdot T \mathrm{~d} x=\int_{\Omega} D_{\mathcal{T}} U \cdot \operatorname{Curl} \phi \mathrm{d} x .
$$

For any continuous and piecewise affine $\Phi$ in $H^{1}(\Omega)$ we have, according to an elementwise integration by parts,

$$
\int_{\Omega} D_{\mathcal{T}} U \cdot \operatorname{Curl} \Phi \mathrm{d} x=-\int_{\cup \mathcal{E}}\left[U\left(\operatorname{Curl} \Phi \cdot n_{E}\right)\right] \mathrm{d} s
$$

where $\left[U\left(\operatorname{Curl} \Phi \cdot n_{E}\right)\right]$ denotes the jump of $U\left(\operatorname{Curl} \Phi \cdot n_{E}\right)$ across the edge $E$ with canonical modifications at the boundary. Assume that $E \in \mathcal{E}$ is an inner edge, $E=T_{1} \cap T_{2}$ for $T_{1}, T_{2} \in \mathcal{T}$. Since $\Phi$ is continuous at $E$ and can be differentiated along $E$ with a jump $[\partial \Phi / \partial s]=0$ on each edge. Moreover, because Curl $\Phi$ is piecewise constant, $\operatorname{Curl} \Phi \cdot n_{E}$ is constant on $E$. Hence,

$$
\int_{E}[U] \operatorname{Curl} \Phi \cdot n_{E} \mathrm{~d} s=\operatorname{Curl} \Phi \cdot n_{E} \int_{E}[U] \mathrm{d} s=0 .
$$

Thus, if we define $\Phi:=\mathcal{I} \phi$ the preceding analysis yields

$$
\int_{\Omega} D_{\mathcal{T}} U \cdot \operatorname{Curl} \Phi \mathrm{d} x=0
$$

Returning to the second term in (6.14), we adopt (6.16) and (6.19) to obtain

$$
\begin{aligned}
-\int_{\Omega}\left(A^{-1} T\right) \cdot(\sigma-\Sigma) \mathrm{d} x & =\int_{\Omega} D_{\mathcal{T}} U \cdot \operatorname{Curl}(\phi-\Phi) \mathrm{d} x \\
& =-\int_{\cup \mathcal{E}}(\phi-\Phi) \cdot[\partial U / \partial s] \mathrm{d} s
\end{aligned}
$$

With $|T|=|\operatorname{Curl} \phi|$ in (6.20), Theorem 3.2 shows

$$
-\int_{\Omega}\left(A^{-1} T\right) \cdot(\sigma-\Sigma) \mathrm{d} x \leq C\left\{\sum_{E \in \mathcal{E}} h_{E}\|J\|_{L^{2}(E)}^{2}\right\}^{1 / 2}\|T\|_{L^{2}(\Omega)} .
$$

With $(6.15)$ and $\|\Sigma-\sigma\|_{L^{2}(\Omega)} \leq \operatorname{Lip}(A)\left\|D_{\mathcal{T}} e\right\|_{L^{2}(\Omega)}$, we eventually obtain

$$
\|T\|_{L^{2}(\Omega)} \leq \operatorname{Lip}(A)\left\|D_{\mathcal{T}} e\right\|_{L^{2}(\Omega)} .
$$

Absorbing $\left\|D_{\mathcal{T}} e\right\|_{L^{2}(\Omega)}$ concludes the proof of Theorem 6.1. 


\section{Application to MiXed Finite ELEMENT Methods}

In this section, we consider a linear model problem for a mixed formulation to approximate $(u, \sigma) \in H_{0}^{1}(\Omega) \times$ $L^{2}(\Omega)^{2}$ that satisfies

$$
\sigma=A \nabla u \quad \text { and } \quad \operatorname{div} \sigma+f=0 \quad \text { in } \Omega
$$

The linear and bounded operator $A: L^{2}(\Omega)^{2} \rightarrow L^{2}(\Omega)^{2}$ is supposed to be be self-adjoint and uniformly elliptic, and we are given $f \in L^{2}(\Omega)$. From standard mixed finite element methods such as Raviart-Thomas (RT), BrezziDouglas-Marini (BDM), or Brezzi-Douglas-Fortin-Marini (BDFM) elements (cf. [7] for details), we obtain an approximation $(U, \Sigma) \in L^{2}(\Omega) \times H(\operatorname{div} ; \Omega)$ that satisfies

$$
\begin{aligned}
\int_{T}(f+\operatorname{div} \Sigma) \mathrm{d} x & =0 \quad(T \in \mathcal{T}) \\
\int_{\Omega} P \cdot Q \mathrm{~d} x+\int_{\Omega} U \operatorname{div} Q \mathrm{~d} x & =0 \quad(Q \in \mathcal{M}) .
\end{aligned}
$$

In (7.2), $\mathcal{T}$ denotes a regular triangulation in the sense of Definition 5.1 with nodes $\mathcal{N}$ and a discrete space $\mathcal{S}$ as in Definition 5.2. In (7.3),

$$
\mathcal{M}:=\operatorname{Curl}(\mathcal{S}) \subseteq H(\operatorname{div} ; \Omega)
$$

is supposed to belong to the trial functions. In (7.3), $P:=A^{-1} \Sigma$ is assumed to be $\mathcal{T}$-piecewise smooth, such that $\operatorname{Curl}_{\mathcal{T}} P \in L^{2}(\Omega)^{2}$, and we may define the jump $[P]$ of $P$ across $E$ (i.e. $[P]=\left.P\right|_{T_{2}}-\left.P\right|_{T_{1}}$ on $E=T_{2} \cap T_{1}$ or $[P]=\left.P\right|_{T}$ on $\left.T \cap \Gamma\right)$ and the tangent unit vector $\tau_{E}$ on $E, \tau_{E} \cdot n_{E}=0$.

The following result sharpens $[1,8]$ (where $R_{z}=0, z \in \mathcal{K}$ ).

Theorem 7.1. There exists a constant $C>0$ which depends on $\max _{T \in \mathcal{T}} h_{T} / \rho_{T}$, A, and the simply connected bounded Lipschitz domain $\Omega$ such that, for all $R_{z} \in \mathbb{R}, z \in \mathcal{N}$, we have

$$
\begin{aligned}
\left\|A^{-1 / 2}(\sigma-\Sigma)\right\|_{L^{2}(\Omega)} \leq & C\left\{\sum_{T \in \mathcal{T}} h_{T}^{2}\|f+\operatorname{div} \Sigma\|_{L^{2}(T)}^{2}\right. \\
& \left.+\sum_{z \in \mathcal{N}} h_{z}^{2}\left\|\operatorname{curl}_{\mathcal{T}} P-R_{z}\right\|_{L^{2}\left(\omega_{z}\right)}^{2}+\sum_{E \in \mathcal{E}} h_{E}\left\|[P] \cdot \tau_{E}\right\|_{L^{2}(E)}^{2}\right\}^{1 / 2} .
\end{aligned}
$$

\section{Remarks 7.1.}

1. For RT, BDM, or BDFM finite elements on $\mathcal{T}$, the condition (7.4) is satisfied on triangles and tetrahedra. For rectangles or quadrilaterals, (7.4) holds for higher order ansatz functions.

2. Since the $H(\operatorname{div} ; \Omega)$-norm is the natural norm for the stress error, the volume contribution $h_{T}^{2} \| f+$ $\operatorname{div} \Sigma \|_{L^{2}(T)}^{2}$ in (7.5) may be regarded of higher order. Indeed, if $\Sigma$ is $\mathcal{T}$-piecewise constant, $\|f+\operatorname{div} \Sigma\|_{L^{2}(T)}^{2} \leq$ $C\|\nabla f\|_{L^{2}(T)}^{2}$.

3. Reverse inequalities hold under some regularity assumptions on the data [8].

4. A posteriori estimates for the displacement error are given in [8].

Proof of Theorem 7.1. Since $A$ is uniformly elliptic, the Lax-Milgram lemma assures the existence of an $a \in$ $H_{0}^{1}(\Omega)$ that satisfies

$$
\int_{\Omega}(A \nabla a) \cdot \nabla \eta \mathrm{d} x=\int_{\Omega}(\sigma-\Sigma) \cdot \nabla \eta \mathrm{d} x \quad\left(\eta \in H_{0}^{1}(\Omega)\right) .
$$


As in the proof of Theorem 6.1 we infer that $b \in H^{1}(\Omega) / \mathbb{R}$ exists with

$$
\sigma-\Sigma=A \nabla a-\operatorname{Curl} b
$$

Hence, integrating by parts and by orthogonality of Curl $b$ to $\nabla u$, we obtain

$$
\begin{aligned}
\left\|A^{-1 / 2}(\sigma-\Sigma)\right\|_{L^{2}(\Omega)}^{2} & =\int_{\Omega}(\sigma-\Sigma) \cdot \nabla a \mathrm{~d} x+\int_{\Omega}(P-\nabla u) \cdot \operatorname{Curl} b \mathrm{~d} x \\
& =\int_{\Omega}(f+\operatorname{div} \Sigma) a \mathrm{~d} x-\int_{\cup \mathcal{E}} b[P] \cdot \tau_{E} \mathrm{~d} s+\int_{\Omega} b \operatorname{curl}_{\mathcal{T}} P \mathrm{~d} x
\end{aligned}
$$

$\left(\left.\operatorname{curl}_{\mathcal{T}} P\right|_{T}=\partial P_{1} / \partial x_{2}-\partial P_{2} / \partial x_{1}\right)$. According to (7.2) and a Poincaré inequality,

$$
\int_{T}(f+\operatorname{div} \Sigma) a \mathrm{~d} x \leq\|f+\operatorname{div} \Sigma\|_{L^{2}(T)} h_{T} c_{T}\|\nabla a\|_{L^{2}(T)},
$$

where $h_{T}=\operatorname{diam}(T)$ and the constant $c_{T}$ depends on the shape of the elements only, in our case, on $\rho_{T} / h_{T}[9]$.

Let $B:=\mathcal{I} b \in \mathcal{S}$ denote the weak interpolant to $b \in H^{1}(\Omega)$ (without any boundary conditions). Then, according to $(7.3,7.4)$,

$$
0=\int_{\Omega} P \cdot \operatorname{Curl} B \mathrm{~d} x
$$

From (7.8-7.10) we deduce

$$
\begin{aligned}
\left\|A^{-1 / 2}(\sigma-\Sigma)\right\|_{L^{2}(\Omega)}^{2} \leq & \left(\max _{T \in \mathcal{T}} c_{T}\right)\left\|h_{\mathcal{T}}(f+\operatorname{div} \Sigma)\right\|_{L^{2}(\Omega)}\|\nabla a\|_{L^{2}(\Omega)} \\
& -\int_{\cup \mathcal{E}}\left[P \cdot \tau_{E}\right](b-\mathcal{I} b) \mathrm{d} s+\int_{\Omega} \operatorname{curl}_{\mathcal{T}} P(b-\mathcal{I} b) \mathrm{d} s .
\end{aligned}
$$

The last two terms are (componentwise) exactly of the form analysed in (3.11), and therefore Corollary 3.1 yields the assertion (7.5) if we notice

$$
\|\nabla a\|_{L^{2}(\Omega)}+\|\nabla b\|_{L^{2}(\Omega)} \leq c\left\|A^{-1 / 2}(\sigma-\Sigma)\right\|_{L^{2}(\Omega)}
$$

with a constant $c>0$ which depends on $\Omega$ and $A$.

\section{REFERENCES}

[1] A. Alonso, Error estimators for a mixed method. Numer. Math. 74 (1996) 385-395.

[2] I. Babuška and W.C. Rheinboldt, Error estimates for adaptive finite element computations. SIAM J. Numer. Anal. 15 (1978) $736-754$.

[3] R. Becker and R. Rannacher, A feed-back approach to error control in finite element methods: basic analysis and examples. East-West J. Numer. Math. 4 (1996) 237-264.

[4] D. Braess, Finite Elements. Cambridge University Press (1997).

[5] D. Braess and R. Verfürth, A posteriori error estimators for the Raviart-Thomas element. SIAM J. Numer. Anal. 33 (1996) $2431-2444$.

[6] S.C. Brenner and L.R. Scott, The Mathematical Theory of Finite Element Methods. Texts Appl. Math. 15, Springer, New-York (1994).

[7] F. Brezzi and M. Fortin, Mixed and hybrid finite element methods. Springer-Verlag (1991).

[8] C. Carstensen, A posteriori error estimate for the mixed finite element method. Math. Comp. 66 (1997) $465-476$.

[9] C. Carstensen and S.A. Funken, Constants in Clément-interpolation error and residual based a posteriori error estimates in Finite Element Methods. Berichtsreihe des Mathematischen Seminars Kiel, Technical report 97-11, Christian-AlbrechtsUniversität zu Kiel, Kiel (1997). 
[10] C. Carstensen and S.A. Funken, Fully reliable localised error control in the FEM. Berichtsreihe des Mathematischen Seminars Kiel, Technical report 97-12, Christian-Albrechts-Universität zu Kiel, Kiel (1997).

[11] C. Carstensen and R. Verfürth, Edge residuals dominate a posteriori error estimates for low order finite element methods. Berichtsreihe des Mathematischen Seminars Kiel, Technical report 97-6, Christian-Albrechts-Universität zu Kiel; SIAM J. Numer. Anal. (to be published).

[12] P. Clément, Approximation by finite element functions using local regularization. RAIRO Anal. Numér. 9 (1975) 77-84.

[13] P.G. Ciarlet, The finite element method for elliptic problems. North-Holland, Amsterdam (1978).

[14] E. Dari, R. Duran, C. Padra and V. Vampa, A posteriori error estimators for nonconforming finite element methods. Math. Modelling Numer. Anal. 30 (1996) 385-400.

[15] K. Eriksson, D. Estep, P. Hansbo and C. Johnson, Introduction to adaptive methods for differential equations. Acta Numer. 4 (1995) 105-158.

[16] V. Girault and P.A. Raviart, Finite Element Methods for Navier-Stokes Equations. Springer, Berlin (1986).

[17] R.H.W. Hoppe and B. Wohlmuth, Element-orientated and edge-orientated local error estimates for nonconforming finite element methods. Math. Modelling Numer. Anal. 30 (1996) 237-263.

[18] R. Temam, Theory and Numerical Analysis of the Navier-Stokes Equations. North-Holland (1977).

[19] R. Verfürth, A review of a posteriori error estimation and adaptive mesh-refinement techniques. Wiley-Teubner (1996).

[20] B. Wohlmuth, Adaptive Multilevel-Finite-Elemente Methoden zur Lösung elliptischer Randwertprobleme. Ph.D. thesis, Math. Inst., TU München (1995).

[21] D. Yu, Asymptotically exact a posteriori error estimators for elements of bi-odd degree. Chinese J. Numer. Math. Appl. 13 (1991) 64-78.

[22] D. Yu, Asymptotically exact a posteriori error estimator for elements of bi-even degree. Chinese J. Numer. Math. Appl. 13 (1991) 82-90. 